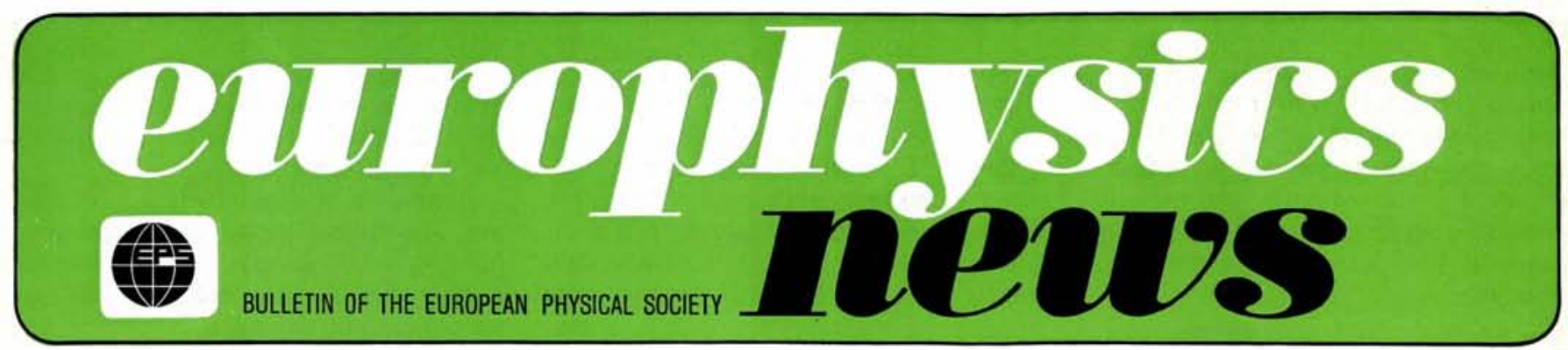

\title{
High Field Superconductors
}

\section{Introduction}

Since the discovery of superconductivity in 1911 by Kamerlingh Onnes, physicists have wanted to apply this extraordinary property in technical projects, especially to build strong electromagnets. The realization of these dreams has in principle been possible for about 15 years, due to the discovery of high field superconductors. Today many physicists and engineers are working on projects where one uses the phenomenon of superconductivity to avoid ohmic losses. Possible large scale applications of superconductivity include among others; generators, motors, magnets for fusion projects, magnetically levitated trains and energy transport. The superconducting materials play in this respect an important role and this article will deal with some aspects of the material problems.

The phenomenon of superconductivity is subjected to several restrictions. The best known of these is the critical temperature $T_{c}$, above which the substance loses its superconducting properties. There are also other limitations. There exists a critical field $\mathrm{H}_{\mathrm{c} 2}$, and a critical current $\mathrm{J}_{\mathrm{c}}$, above which the normal state properties are restored. One finds furthermore that in general the superconducting properties deteriorate with mechanical strain or neutron bombardment. This last limitation will be of importance in the future when superconductivity is applied in the production of energy by fusion.

In the last 15 years much effort has been put into the search for higher $T_{c}$ materials. The reason is obvious; in all applications one would be happy to be able to work at higher temperatures. As a result $T_{c}$ is increasing slowly but steadily and the highest $T_{c}$ known today is the $23.4{ }^{\circ} \mathrm{K}$ of $\mathrm{Nb}_{3} \mathrm{Ge}$ prepared by a special deposition technique.

The other limitations, of $\mathrm{H}_{\mathrm{c} 2}$ and $\mathrm{J}_{\mathrm{c}}$, have received somewhat less attention, probably because physicists say that if one can increase $T_{c}$, other properties will be improved with it. This might be true in some cases, but if we compare compounds with relatively small differences in $T_{c}$ it will not always be true that the compound with the higher $T_{c}$ has the higher $\mathrm{H}_{\mathrm{c} 2}$. Since so far physicists have only been able to increase $T_{c}$ by small steps it seemed reasonable to put more effort into the study of one of the other parameters. This report is concerned with the upper critical field $\mathrm{H}_{\mathrm{e} 2}$, the reason being that recently a new class of materials, the ternary molybdenum sulfides, has been found which exhibit much higher critical fields than those found so far in other compounds.

\section{Why is there a critical field ?}

Before we go on to discuss the recent results, we should ask ourselves why a magnetic field destroys superconductivity. Why do we have a critical field?

The successful recipe used by Bardeen, Cooper and Schrieffer (BCS) ${ }^{1}$ in their theory of superconductivity was to construct the ground state from pairs of free electrons. These pairs were of the type $|k, \uparrow\rangle|-k, \downarrow\rangle$, where $k$ is the wave vector of the electron and the arrows indicate the spin states. One essential property of these two states is that they are degenerate. In a more realistic model where for instance impurity potentials are present, Anderson has pointed out ${ }^{2}$ that the two electron states in a pair should be chosen as time reversed states $\left|\psi_{n}\right\rangle$, $\mathrm{C}\left|\psi_{\mathrm{n}}\right\rangle$, where $\left|\psi_{\mathrm{n}}\right\rangle$ is an eigen function of the one electron Hamiltonian and $\mathrm{C}$ is the time reversal operator. The necessary condition for a BCS type of superconducting state is that the Hamiltonian $\mathcal{H}$ commutes with C. This means that the two states $\left\langle\psi_{n}\right\rangle$ and

\section{O. Fischer, Geneva}

$\mathrm{C} \psi_{n}>$ are degenerate. This pairing concept has proved to be quite successful in superconductors. Every time the Hamiltonian contains terms which do not commute with $\mathrm{C}$ one finds the so-called pair breaking effects. Qualitatively one may say that the term that does not commute with $C$ treats the two electron states in a pair differently, the degeneracy is lifted and this leads to a destruction of the state which is built up of these pairs. A well known example for such pair-breaking effects is the influence of magnetic impurities on the critical temperature of a superconductor.

In the presence of a magnetic field $\mathrm{H}$ the Hamiltonian of a free electron is

$\mathcal{H}=\frac{1}{2 m}\left(\vec{p}-\frac{e}{c} \vec{A}\right)^{2}-g \mu_{B} \overrightarrow{\sigma H}$

where $\vec{A}$ is the vector potential and $\vec{\sigma}$ the electron spin. It is easy to see that $\overrightarrow{\mathrm{pA}}$ as well as $\overrightarrow{\sigma \mathrm{H}}$ change sign under time reversal. Thus $\mathcal{H}$ does not commute with $\mathrm{C}$. Both terms produce pairbreaking and lead to a critical field.

Let us first discuss the right hand term. It is easy to see why this should lead to pair-breaking. The pairing concept tells us that we should pair states with opposite spins. The magnetic field will produce a Zeeman-splitting of the two states in the pair so that they are no longer degenerate and

\section{Contents}

High Field Superconductors Letter to the Editor

APS Award to Professor Dr. H. Welker

3rd General Assembly of EPS Members

3rd European Meeting on Ferroelectricity (EMF-3)

Advisory Committee on Physics Education

Individual Ordinary Members

Evidence for Charm

Europhysics News is published monthly by the European Physical Society. (C) 1976. Reproduction rights reserved. 
this will lead to a destruction of the superconducting state. Expressed differently, the magnetic field will try to align the spins of the two electrons in a pair and thus destroy the pair. For a BCS superconductor this interaction leads at $T=0$ to a first order transition at 3

$$
\mathrm{H}_{\mathrm{po}}=18.4 \mathrm{~T}_{\mathrm{c}} \text { (k gauss) }
$$

$\mathrm{H}_{\mathrm{po}}$ is often referred to as the Clogston limit. Thus the interaction of the external field with the spins of the conduction electrons alone will lead to a critical field $\mathrm{H}_{\mathrm{po}}$ which is $184 \mathrm{k}$ gauss for a superconiductor with $\mathrm{T}_{\mathrm{c}}=10^{\circ} \mathrm{K}$.

However, most superconductors with $\mathrm{T}_{\mathrm{c}}$ of the order of $10^{\circ} \mathrm{K}$ have a much lower critical field than $184 \mathrm{k}$ gauss. This means that the influence of the magnetic field on the orbits of the superconducting electrons, described by the first term in equation (1), is the dominant interaction in most superconductors. For most superconductors we may then ignore the second term in eq. (1) and only take into account the influence of the magnetic field on the orbits.

The discussion of the orbital effects may be done very conveniently by using the Ginsburg-Landau equations, which can be derived from the microscopic theory ${ }^{4}$. We will not enter here into the details of these equations, but merely recall that they contain two characteristic lengths; $\lambda$, the penetration depth and $\xi$, the coherence length. $\lambda$. may be considered as the minimum distance over which the magnetic field may show important variations in the superconductor and $\xi$ is the distance necessary for the superconductivity to be restored if there is a normal region at any place in the material.

Two quite different situations arise depending whether $\gamma^{2} \lambda / \xi$ is larger or smaller than one. If $\gamma^{2} \lambda / \xi<1$ we have a type I superconductor. These superconductors show a complete Meissner effect i.e. the magnetic field is excluded from the interior of the material, only penetrating a distance $\lambda$ into the superconductor. Due to this exclusion of the field there is a large difference in the magnetic free energy between the superconducting state and the normal state. This leads to a first order transition to the normal state at a relatively low field $\mathrm{H}_{\mathrm{c}}$ typically of the order of a few hundred gauss.

A quite different situation arises if $V^{2} \lambda / \xi>1$. In this case the surface energy between a normal and a superconducting region is negative in the presence of a magnetic field. The system therefore prefers, as soon as the field exceeds a certain limit $\mathrm{H}_{\mathrm{cl}}\left(<\mathrm{H}_{\mathrm{c}}\right)$, to have a large surface area between normal regions and superconducting regions. The normal regions are realized in the form of fluxlines. These are filamentary normal regions in the direction of the magnetic field. The material is completely normal only in the centre of the line but a distance $\xi$ is needed to restore the superconducting state, one therefore speaks of the fluxline as having a diameter $2 \xi$. The magnetic field penetrates the sample through the normal region in the centre of the fluxline and since in a typical type II superconductor we have $\lambda>>\xi$, the magnetic field will penetrate far into the superconducting region. As a consequence the system may remain superconducting up to fields much higher than $\mathrm{H}_{\mathrm{c}}$. When the external field is increased the number of fluxlines increases so that the mean value of the field increases. At a field $\mathrm{H}_{\mathrm{c} 2}$ the fluxlines completely fill the material and the field in the interior equals the external field. At this field the system makes a second order transition to the normal state. The resulting magnetization curve is shown in Fig. 1.
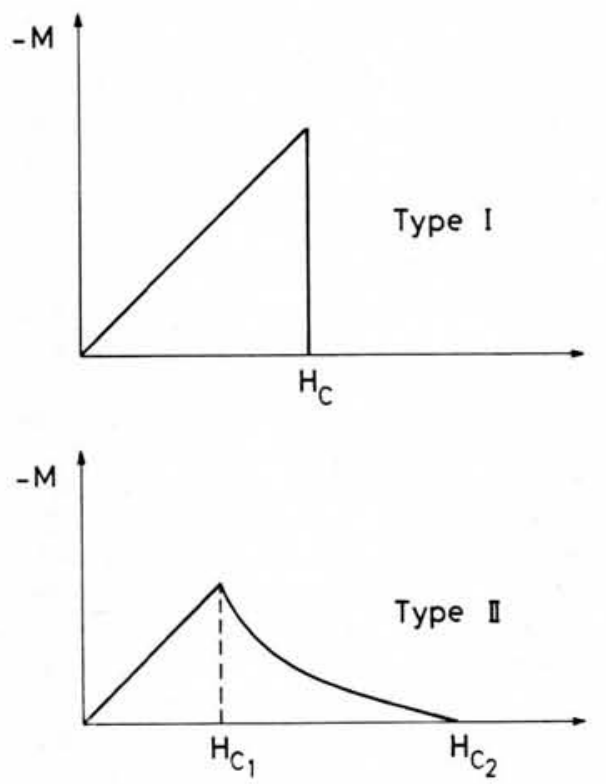

Fig. 1 Magnetization Curves for a type $/$ and a type II superconductor

It is important to note that although the magnetization gradually goes to zero as one increases $\mathrm{H}$ towards $\mathrm{H}_{\mathrm{e} 2}$, the static resistivity remains zero up to $\mathrm{H}_{\mathrm{c} 2}$, where it abruptly changes to the normal state resistivity. Thus, from the point of view of applications it is important to know what are the possible values of $\mathrm{H}_{\mathrm{c} 2}$.

The interest in high critical fields is not only technical. It is obvious from the above that the existence of a critical field is connected with the type of pairing. If for some reason nature should choose another sort of pairing the influence of a magnetic field may not be the same.

Examples of different types of pairing are found today in "Superconducting" $\mathrm{He}^{3}$, so that it is not a completely utopian idea that in some superconducting compound one might also find the same thing.

\section{How to obtain high critical fields ?}

It can be shown that the total flux associated with one flux line i.e. the integral of the field over a cross-section of the line, is exactly one flux quantum $\Phi_{0}=2.0710^{-7}$ gauss $\mathrm{cm}^{2}$. Above we saw that at $\mathrm{H}_{\mathrm{e} 2}$ the fluxlines of diameter $2 \xi$ fill the material completely. Thus the critical field must be of the order $\Phi_{0} / 2 \pi \xi^{2}$. A high critical field therefore means a small coherence length $\xi$.

Let us take as an example pure niobium. It has a $T_{c}$ of $9.2^{\circ} \mathrm{K}$. Theory tells us that in such a pure system $\xi=0.13 \mathrm{hv}_{\mathrm{F}} / \mathrm{k}_{\mathrm{B}} \mathrm{T}_{\mathrm{c}}$. Here $\mathrm{v}_{\mathrm{F}}$ is the Fermi velocity i.e. the velocity of an electron at the Fermi-surface. What is the order of magnitude of $\xi$ ? Typical values of $\mathrm{V}_{\mathrm{F}}$ are $10^{7} \mathrm{~cm} / \mathrm{sec}$ to $10^{8} \mathrm{~cm} / \mathrm{sec}$, and if we take $T_{\mathrm{c}} \approx 10^{\circ} \mathrm{K}$ we find that $\xi$ is typically several hundred Ångströms. If we substitute this into the formula for $\mathrm{H}_{\mathrm{e} 2}$ we find a critical field of the order of one kilogauss. The theory then predicts that a pure system such as $\mathrm{Nb}$ should have a low critical field of at maximum a few $k$ gauss. This is also what is found. $\mathrm{Nb}$ is a type II superconductor with a critical field of about $2.5 \mathrm{k}$ gauss.

This, however, is far from what we would like to have as a critical field, and so the question remains: How can we get higher critical fields? The answer to this question was found by studying alloys. If we alloy $\mathrm{Nb}$ with say $\mathrm{Ti}$, there is, up to high concentration of $\mathrm{Ti}$, no important change in $T_{c}$ or $v_{F}$ There is however an increase of about a factor 50 in the critical field. The main difference between $\mathrm{Nb}$ and $\mathrm{NbTi}$ is found in the mean free path, which is the mean distance over which the electron can travel in the normal state without being scattered by impurities or by inhomogeneities.

$\mathrm{Nb}-\mathrm{Ti}$ is very "dirty", meaning that it is very disordered, and this leads to a very short $A$. Thus from experiments we would expect $\xi$ to depend on $\ell$, and in fact, if we calculate $\xi$ for a dirty superconductor $(\xi>>\ell)$ we find $\xi=0.36\left(\mathrm{hv}_{\mathrm{F}} / \mathrm{kT} \mathrm{c}_{\mathrm{c}} \ell\right)^{1 / 2}$ In general a dirty material will therefore have a higher critical field than a pure material. Or, 
expressed differently; a high field superconductor will in general be a very bad conductor in the normal state. This is seen directly if we express the normal state resistivity $\varrho$ in terms of $\ell$. One then finds that $\mathrm{H}_{\mathrm{c} 2} \sim \mathrm{oT}_{\mathrm{c}}$. For $\mathrm{NbTi} \ell$ is of the order of $10 \AA$ and hence this explains the very high values of $\mathrm{H}_{\mathrm{e} 2}$.

In dirty systems, such as we have discussed above, in general the critical field reaches values of the order of $100 \mathrm{k}$ gauss. These are already quite high fields and in most applications of superconductivity one today uses wires of such materials. This is not however the attainable limit. In systems where both $v_{F}$ and $\ell$ are small $\xi$ may become extremely small. For $v_{F}$ and $A$ both to be small, the conduction electrons should have a localized character. Therefore we may expect very high critical fields in quasi low - dimensional systems. By low - dimensional systems we mean those in which the overlap between atomic wave functions is small in one or several space directions.

\section{Recent results}

Before discussing results we should perhaps say something about how one produces the high magnetic fields necessary to measure $\mathrm{H}_{\mathrm{c} 2}$. Today static magnetic fields may be obtained up to $150 \mathrm{k}$ gauss with superconducting solenoids or about $200-230 \mathrm{k}$ gauss with the water cooled Bittertype magnets in one of the larger magnet laboratories. Fields of $300 \mathrm{k}$ gauss and above can so far only be achieved by pulsed techniques. All measurements at very high fields reported here have therefore been measured in slowly pulsed magnetic fields. The highest "slow field" made in Geneva is $600 \mathrm{k}$ gauss with a pulse time of $7.5 \mathrm{msec}$.

We now turn to the more recent results.

The most striking quasi two - dimensional superconducting class of materials is formed by the layer-compound of the type $\mathrm{NbSe}_{2}$. Here the structure contains layers approximately $6 \AA$ thick, which are coupled together by weak van der Waals forces. The electronic properties of these compounds are extremely anisotropic. This anisotropy is also seen in the critical field. If the external field is perpendicular to the layers, Fig. 2, then the screening current in the fluxline remains within the layers without crossing the boundary between adjacent layers (this current is of course perpendicular to the field). Within each layer the Fermi velocity and $\ell$ have normal values and so the critical field

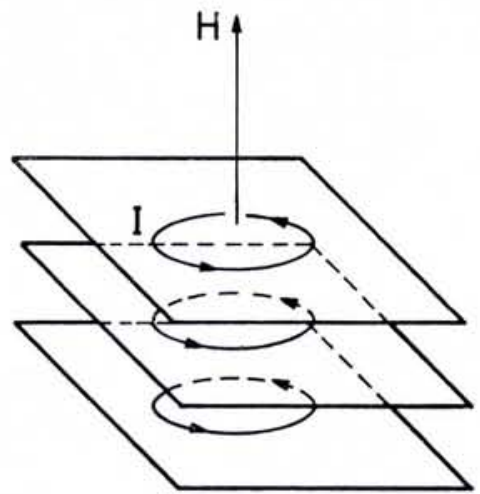

Fig. 2 Schematic illustration of the difference between a parallel and a perpendicular magnetic field on a layer structure

is rather low. However, if the field is parallel to the layers, the screening currents, which are still in a plane perpendicular to the external field, will also be in a plane perpendicular to the layers and so the coherence length perpendicular to the layers $\xi \perp$, will enter the expression for $\mathrm{H}_{\mathrm{e} 2}$. Since the coupling is very weak between the layers, $\xi \perp$ will be very small for the reasons described above and therefore $\mathrm{H}_{\mathrm{c} 2}$ will be very high. A particularly interesting situation arises in these compounds since they can be intercalated by organic molecules, like pyridine. This separates the layers by $6 \AA$ or more so that the coupling becomes weaker, but without destroying superconductivity. In $\mathrm{TaS}_{2}$ intercalated with pyridine $T_{c}$ is about $3.5^{\circ} \mathrm{K}$ thus rather low, but in spite of this the upper critical field parallel to the layers is above $200 \mathrm{k}$ gauss $\mathrm{s}^{5,6}$. The ratio $\mathrm{H}_{\mathrm{c} 2} / \mathrm{T}_{\mathrm{c}}$ is now much higher than in $\mathrm{Nb}-\mathrm{Ti}$, and the anisotropy in $\mathrm{H}_{\mathrm{e} 2}$ exceeds a factor of 10 ! These compounds therefore demonstrate clearly that high critical fields mean "localized conduction electrons" or quasi low - dimensional systems.

Another class of materials with high critical fields are the superconductors with the A-15 structure of composition $\mathrm{A}_{3} \mathrm{~B}$. Examples are $\mathrm{Nb}_{3} \mathrm{Sn}, \mathrm{Nb}_{3} \mathrm{Ge}, \mathrm{V}_{3} \mathrm{Si}$, $\mathrm{V}_{3} \mathrm{Ga}$ and others.

It is known that in most of these compounds superconductivity is due to the d-electrons of the A-atoms localized on linear chains, and that this linear chain structure is responsible for the excellent superconducting properties of these compounds. However one cannot speak of these compounds as low-dimensional in the same sense as in the layer-compounds and thus $\mathrm{H}_{\mathrm{c} 2} / \mathrm{T}_{\mathrm{c}}$ is typically a factor of two lower in the former. However these compounds have the highest $T_{c}$ values known today and so $\mathrm{H}_{\mathrm{c} 2}$ may nevertheless become very high. For $\mathrm{T}_{\mathrm{c}} \approx 21^{\circ} \mathrm{K}$ critical fields of above $400 \mathrm{k}$ gauss have

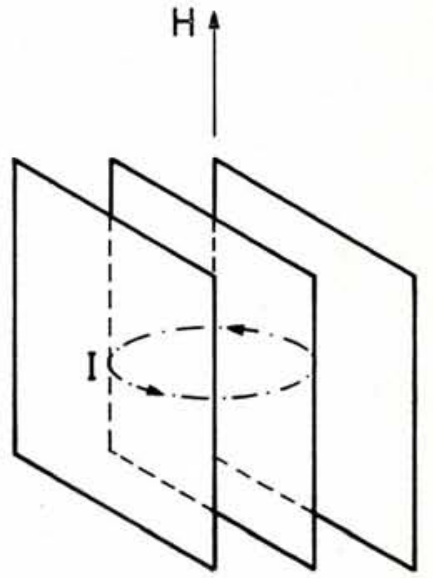

been found in $\mathrm{Nb} .79 \quad\left(\mathrm{Al}{ }_{73} \mathrm{Ge}{ }_{.27}\right)_{.21}{ }^{7}$ whereas $\mathrm{Nb}_{3} \mathrm{Ge}$ which has the highest $\mathrm{T}_{\mathrm{c}}\left(\approx 23.2^{\circ} \mathrm{K}\right)$ has a critical field of about $380 \mathrm{k}$ gauss ${ }^{8}$.

As mentioned in the introduction, there has recently been new progress in the search for even higher critical field superconductors. A new class of materials with the general formula $\mathrm{M}_{\mathrm{y}} \mathrm{Mo}_{6} \mathrm{X}_{8}$ has been found. $\mathrm{M}$ stands for a variety of different metals (Examples are $\mathrm{Pb}, \mathrm{Sn}, \mathrm{Cu}, \mathrm{Yb}, \mathrm{La}, \mathrm{Gd}$, $\mathrm{Eu}, \mathrm{Mn}, \mathrm{Cr}, \mathrm{Fe}$, and others) and $\mathrm{X}$ is either Sulphur, Selenium or Tellurium. Most of these compounds are superconducting with very high $\mathrm{H}_{\mathrm{e} 2} / \mathrm{T}_{\mathrm{c}}$ ratios. These compounds were first described by three French chemists Chevrel, Sergent and Prigent $^{9}$ and soon thereafter Matthias and coworkers ${ }^{10}$ found superconductivity in several of these compounds, whereas their high critical fields were discovered one year later in Geneva ${ }^{11}$. The highest $T_{c}$ value in this class is about $15^{\circ} \mathrm{K}$ and is found in $\mathrm{PbMo}_{6} \mathrm{~S}_{8}$. Critical fields of the order of $600 \mathrm{k}$ gauss $12,13,14$, have been found in this material and it is even possible to increase the critical field somewhat by replacing some of the $\mathrm{Pb}$ atoms by magnetic $\mathrm{Eu}$ atoms. In this way the critical field is pushed up towards $700 \mathrm{k}$ gauss ${ }^{15}$.

In Fig. 3 are shown the critical fields versus temperature for the four classes of materials we have been discussing. We see that $\mathrm{H}_{\mathrm{c} 2} / \mathrm{T}_{\mathrm{c}}$ is about the same for the dirty systems like $\mathrm{NbTi}$ and the $\mathrm{A}-15$ type materials. The latter ones have higher critical fields because of their higher critical temperatures. The values of $\mathrm{H}_{\mathrm{c} 2} / \mathrm{T}_{\mathrm{c}}$ for the layer-compounds and the ternary molybdenum chalcogenides are much higher. In the layer-compounds this is clearly due to the quasi two-dimensional structure. What is the reason in the $\mathrm{M}_{\mathrm{y}} \mathrm{Mo}_{6} \mathrm{X}_{8}$ compounds?

The structure of these compounds is shown in Fig. $4^{16,17}$. The structure 


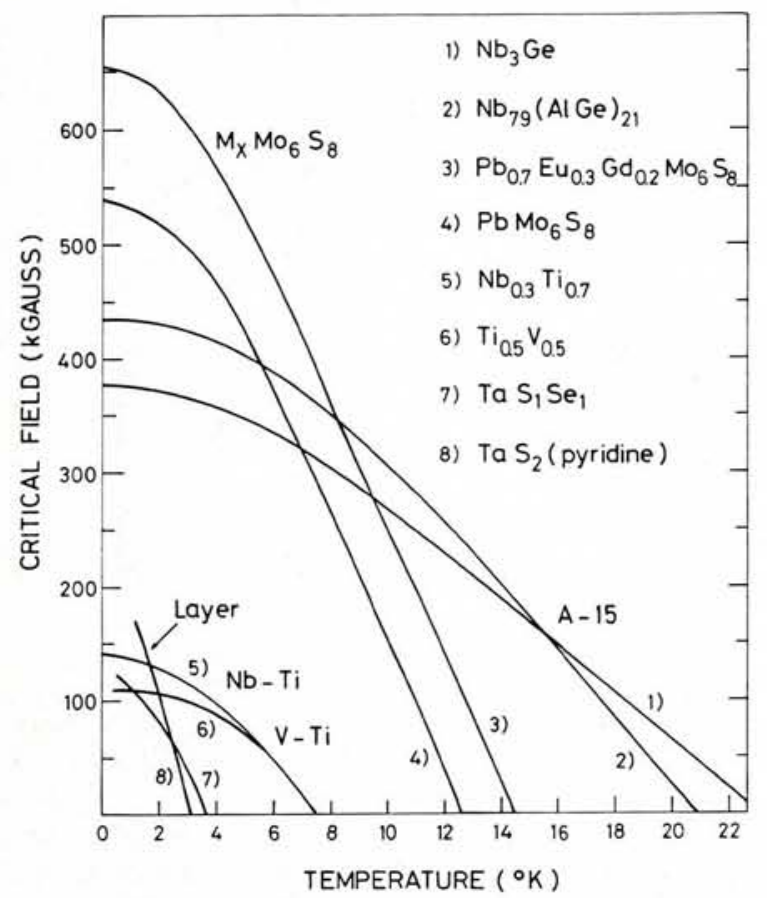

Fig. 3 Comparison between different high field superconductors

contains octahedrons of 6 Mo atoms and these octahedrons are well separated from each other in space. Different experimental results, especially those where the element $M$ is magnetic, point to the result that superconductivity is due to the d-electrons of Molybdenum localized on the Mo-octahedrons. Since these octahedrons are separated from each other in space, we expect the overlap of wavefunctions to be small and so we are in fact in a quasi low-dimensional system. In this case it is a quasi "zero-dimensional" system since in all three space directions the coupling is presumed to be weak.

\section{Speculations on future developments}

In most developments of large scale applications of superconductivity today one uses $\mathrm{Nb}-\mathrm{Ti}$ wires. This means that one has to work with fields well below $100 \mathrm{k}$ gauss. Multifilament $\mathrm{Nb}_{3} \mathrm{Sn}$ wires are now also available and they allow the field to be increased to about $150 \mathrm{k}$ gauss and it is expected that with $\mathrm{V}_{3} \mathrm{Ga}$ wires one will be able to approach $200 \mathrm{k}$ gauss.

However, a quick glance at fig. 3 tells us that there is a large gap between the highest observed critical fields and the fields that can be produced by using superconductors. Thus it should be possible to produce static fields much higher than we can reach today; - say of the order of 400 to $500 \mathrm{k}$ gauss. How fast we can reach these high fields will certainly depend on the real need for higher static fields, since the development of ultrahigh field superconducting wires will meet a lot of technical problems. One example is the influence of the huge forces in an ultrahigh field magnet on the properties of the superconducting material itself. A domain where ultrahigh magnetic fields may be needed is in the possible production of energy by fusion, whereas in certain other applications the gain by going to higher fields may seem less evident.

From the point of view of the materials another question is of interest: Have we arrived, with the $\mathrm{M}_{\mathrm{y}} \mathrm{Mo}_{6} \mathrm{X}_{8}$ materials, at an upper limit of the critical fields? If we compare the observed $\mathrm{H}_{\mathrm{c} 2}$ with the predicted paramagnetic critical field for a BCS superconductor (Clogston limit) which we discussed the beginning of this article we find that, both for the
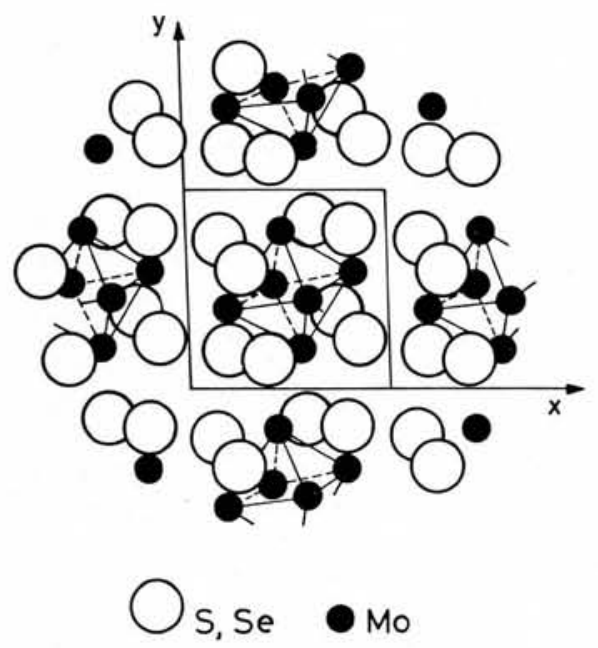

Fig. 4 Representation of the structure of $\mathrm{MO}_{3} \mathrm{~S}_{4}$ and MosSes. In M Mos $\mathrm{S}_{8}$ the M-elements enter into the channels between the Mooctahedrons layer-compounds as well as for the ternary molybdenum chalcogenides the measured critical field is more than twice $H_{p 0}$. This unexpected discrepancy has brought many physicists to believe that a type of pairing other than the conventional BCS-Anderson one takes place in these compounds. However we will not enter into a discussion of this subject here since there is no experimental confirmation apart from the anomalously high critical fields. One thing that is sure, is that the Clogston limit is not a real limit in these materials and it seems as if the orbital effects are also the more important ones here. If this is true then the coherence length $\xi$ can be estimated from $\mathrm{H}_{\mathrm{c} 2}$ using the simple relation

$$
\mathrm{H}_{\mathrm{e} 2}=\Phi_{\mathrm{o}} / 2 \pi \xi 2 \text {. }
$$

For the highest $\mathrm{H}_{\mathrm{c} 2}$ of about $700 \mathrm{k}$ gauss, we find $\xi \approx 20 \AA$. This distance corresponds to three octahedrons of $\mathrm{Mo}_{6}$. There seems to be no reason that just this particular distance should constitute an absolute limit on the smallness of $\xi$. Within the model given above for these compounds one would expect that the diameter of one cluster would constitute a lower limit on $\xi$. This distance is only one third of the observed $\xi$ thus there seems to be no obvious reason that one cannot get even much higher critical fields in compounds of this type.

In conclusion, we note that although the highest critical fields known today are so high that we meet serious difficulties when we want to measure them, it seems quite probable that in the future, compounds with even higher critical fields will be found.

\section{REFERENCES}

1. J. Bardeen, L. N. Cooper and J. R. Schrieffer,

2. P.W. Wev. Anderson, J. Phys. Chem. Solids 11, 26

3. A. M. Clogston, Phys. Rev. Letters 9, 266 (1962) B. S. Chandrasekhar, Appl. Phys. Letters 1, 7

4. For a review of the Ginzburg-Landau theory see for instance: St James, Sarma and Thomas: "Oxford, 1969 .

press Oxtord, ang

C. V. Coleman, Phys. Rev,

B. Foner and E. J. McNiff, Phys. Letters 45A, $429(1973)$
S. Foner,

E J. McNiff, B. T. Matthias, T, H. Geballe, R. H. Willens and E. J. Corenzwit, Phys. Letters 31A, 349 (1970)

8. S. Foner, E. J. McNiff, J. Gavaler and M. Janocko, Phys. Letters 47A, 485 (1974)

9. R. Chevrel, M. Sergent and J. Prigent, J. Sol. State Chem. 3, 515 (1971)

10. B. T. Matthias, M. Marezio, E. Corenzwit, A.S Cooper and H. E. Barz, Science 175, 1465 (1972) 1. R. Odermatt, Ø. Fischer, H. Jones and G. Bongi, J. Phys. C 7, L13 (1974)

12. S. Foner, E. J. MCNiff and E. J. Alexander, Phys. Letters 49A, 269 (1974)

13. Ø. Fischer, H. Jones, G. Bongi, M. Sergent and R. Chevrel, J. Phys. C 7, L450 (1974)

14. $\phi$. Fischer, Proceedings of the fourteenth International Conference on Low Temperature Physics, Helsinki, August 1975

15. Ø. Fischer, M. Decroux, S. Roth, R. Chevrel, and M. Sergent, J. Phys. C 8, L474 (1975)

. Marezio, . Dernier, Mat, Res. Bull 8 . Corenzwit and B. Matthias, Mat. Res. Bull. 8,
657 (1973)

17. J. Guillevic, Thèse, University of Rennes 1974 